\title{
Overexpression of Selenoprotein H Reduces Ht22 Neuronal Cell Death aiter UVB Irradiation by Preventing Superoxide Formation
}

\author{
Kamel E. Ben Jilani, Jun Panee, Qingping He, Marla J. Berry, and Ping-An Li \\ Department of Cell Molecular Biology, John A. Burns School of Medicine, University of Hawaii, Honolulu, HI 96813, USA
}

Correspondence to: Dr. Kamel E. Ben Jilani, Department of Cell Molecular Biology, John A. Burns School of Medicine, 651 Ilalo Street, BSB 217, Honolulu, HI96813, USA; E-mail: kamel@hawaii.edu

Received: 2006.11.27; Accepted: 2007.02.05; Published: 2007.02.11

Selenoproteins have been shown to exhibit a variety of biological functions, including antioxidant functions, maintaining cellular redox balance, and heavy metal detoxification. UV irradiation-induced damage is partially mediated by increased oxygen radical production. The present study is designed to examine the antioxidative effects of human selenoprotein $\mathrm{H}(\mathrm{hSelH})$ after brief period of UVB irradiation on the murine hippocampal neuronal cell line Ht22. Ht22 cells were stably transfected with the hSelH gene or with MSCV empty vector and exposed to UVB irradiation with or without the presence of serum. The results showed that cell viability was significantly higher in hSelH-transfected cells compared to the MSCV vector-transfected cells after $24 \mathrm{~h}$ of recovery with or without the presence of serum in the media. Further studies revealed that while the number of superoxide anion $\left(\mathrm{O}^{\circ}-\right)$ positive cells was increased following a $7 \mathrm{~mJ} / \mathrm{cm}^{2}$ of $\mathrm{UVB}$ irradiation and $5 \mathrm{~h}$ of recovery, overexpression of hSelH significantly reduced superoxide production. These results suggest that hSelH overexpression protects cells from UVB irradiation-induced cell death by reducing the $\mathrm{O} 2$ - formation.

Key words: selenoprotein H; ultraviolet B irradiation; reactive oxygen species; superoxide anion; cell viability.

\section{Introduction}

Ultraviolet (UV) irradiation, especially UVB irradiation (290-320 $\mathrm{nm})$, leads to premature skin aging, suppression of immunity, and skin cancer [1]. In neuronal cells, UV irradiation induces apoptosis; therefore, many research groups have used this model as a tool to study the intracellular mechanisms of apoptosis [2-7]. UV irradiation induces cell death via a variety of cellular mechanisms. These include induction of nuclear DNA damage, activation of cell surface death receptors such as the tumor necrosis factor (TNF) superfamily, and formation of reactive oxygen species (ROS) accompanied by mitochondrial dysfunction and release of pro-apoptotic factors $[8,9]$. UV irradiation has been demonstrated to produce reactive oxygen species (ROS) such as superoxide anion $\left(\bullet \mathrm{O}_{2}\right)$ radical, hydroxyl radical, hydrogen peroxide and the highly reactive and destructive oxidant, peroxynitrite (ONOO-) [8-10].

To date, 25 selenoprotein genes have been identified in human. Known biological roles of selenoproteins include antioxidant enzymes, enzymes involved in thyroid hormone metabolism, and proteins functioning in selenium transport and heavy metal detoxification [11-16]. These include several well-known peroxidases and reductases such as glutathione peroxidases (GPxs), phospholipid hydroperoxide glutathione peroxidases (PHGPXs) and thioredoxin reductases (TRs), as well as other lesser characterized selenoproteins such as selenoprotein $\mathrm{H}$ (SelH) [17, 18]. Furthermore, selenoprotein P (SelP) may also function as a neural survival factor [19, 20]. Emerging evidence suggests that SelP may also possess antioxidant activity [21, 22]. For example, SelP has been shown to function as a protectant against diquat-induced liver necrosis and lipid peroxidation in the rat $[23,24]$. SelP is also protective against peroxynitrite-mediated oxidation and nitration [25]. The antioxidative effects of several other selenoproteins such as Gpx, PHGPXs and TRs have been well characterized [26, 27].

The function of SelH is largely unknown. Recently, it was demonstrated that overexpression of Drosophila melanogaster SelH (dSelH) significantly increased viability in embryos by decreasing lipid peroxidation [28]. The objectives of this study were to investigate whether human Selenoprotein $\mathrm{H}$ (hSelH) protects neuronal cells against UVB irradiation-induced cell death and if so, whether the protective effect may associate with ROS production prior to observable cell death. We first determined cell viability following a $7 \mathrm{~mJ} / \mathrm{cm}^{2}$ of UVB irradiation injury and $24 \mathrm{~h}$ of recovery in the murine hippoccampal neuronal cell line Ht22, transfected with the hSelH gene in Murine Stem Cell Virus (MSCV) or the empty retroviral vector. We then measured $\mathrm{O} 2$-production after $5 \mathrm{~h}$ of recovery in $\mathrm{hSelH}$ or empty vector transfected cells using the oxidized dihydroethidine detection method $[29,30]$. Our results are consistent with the concept that the neuroprotective effect of SelH is associated with its ability to reduce ROS production. 


\section{Materials and Methods}

\section{hSelH transfection procedures}

The plasmid containing the gene encoding hSelH was purchased from Invitrogen (Carlsbad, CA, USA). The target gene was subcloned into Murine Stem Cell retroviral vector pMSCVpuro, purchased from $\mathrm{BD}$ Biosciences (Palo Alto, CA, USA). The packaging cells RetroPack PT67 (BD Biosciences) were plated in a 60 $\mathrm{mm}$ plate at $70 \%$ optical confluency $12 \mathrm{~h}$ before transfection. Cells were then transfected with $2 \mu \mathrm{g}$ of hSelH-carrying retroviral vector or empty vector using Fugene method (Roche, Indianapolis, IN, USA). Culture medium was aspirated from cultures after $4 \mathrm{~h}$ of transfection. PT 67 cells were washed twice with PBS, and grown for $24 \mathrm{~h}$ in $3 \mathrm{ml}$ complete medium. The cells were then selected by puromycin. The concentration of puromycin was optimized to kill the untransfected control PT67 cells in 7-10 d. The transfected PT67 cells that survived the puromycin selection stably produced virus. For viral infection of the target cells, Ht22 cells were plated $12 \mathrm{~h}$ before infection at $\sim 70 \%$ optical confluency. The medium from transfected PT67 cells containing virus were collected, filtered through a $0.45 \mu \mathrm{m}$ filter, and added to the Ht22 cells in the presence of $4 \mu \mathrm{g} / \mathrm{ml}$ Polybrene. The virus-containing medium was replaced after $24 \mathrm{~h}$ of incubation. After infection for $48 \mathrm{~h}$, the target cells were subjected to puromycin selection. The selection lasted for $\sim 10 \mathrm{~d}$ until the uninfected control cells were killed. The cells that survived the selection made up the hSelH-transfected Ht22 cell line (hSelH-Ht22) and MSCV-transfectant (MSCV-Ht22) cell lines.

\section{Detection of mRNA overexpression on hSelH by Real time PCR}

Total RNA was extracted from hSelH-Ht22 and MSCV-Ht22 cells using RNeasy Midi Kit (Qiagen, Valencia, CA, USA). cDNAs were synthesized from total RNA using the SuperScript III First-Strand Synthesis System (Invitrogen), and PCR amplification of target genes were carried out using the Platinum SYBR Green qPCR SuperMix-UDG kit (Invitrogen). The following specific primers were used to amplify hSelH, mSelH, and mGAPDH (house keeping gene) cDNAs:

$$
\text { hSelH }
$$

(5'-GCTTCCAGTAAAGGTGAACCCG-3') hSelH

(5'-ACCCAAATCTCCCTACGACAGG-3') mSelH

(5'-GGAAGAAAGCGTAAGGCGGG-3') mSelH

(5'-GGTTTGGACGGGTTCACTTGC-3') MGAPDH (5'-TGACATCAAGAAGGTGGTGAAGC-3')
and mGAPDH

(5'-CCCTGTTGCTGTAGCCGTATTC-3')

All PCR reactions were performed in duplicate 20 $\mu 1$ reaction volumes and detection was carried out with a LightCycler 2 real-time PCR machine (Biorad,
Hercules, CA, USA).

\section{Cell maintenance and treatment}

Murine hippocampal Ht22 neuronal cells were infected either with the MSCV empty vector or with $\mathrm{hSelH}$ in MSCV expression vector. For propagation, cells were fed with Dulbecco's Modified Eagle Medium (DMEM) with 10\% fetal bovine serum (FBS), $2 \mathrm{mM}$ glutamine, and $200 \mathrm{mM}$ streptomycin/penicillin (Invitrogen) and then cultured at $70 \%-75 \%$ relative humidity in $5 \% \mathrm{CO}_{2}$ at $37^{\circ} \mathrm{C}$. Media was renewed every $3 \mathrm{~d}$. For cell viability assays, cells were seeded in 48-well cell culture plates (Corning, Aton, MA, USA) $48 \mathrm{~h}$ before treatment. The cells were allowed to reach $80 \%$ optical confluency before UVB treatment.

\section{UVB irradiation}

Cells were seeded in 96 or 24 wells plate and propagated to reach $80 \%$ cell confluency. Prior to UVB irradiation, cells were washed twice with cold PBS to remove residual serum and dead cells. Cells were placed in serum-containing media $(10 \%)$ media or without serum for $1 \mathrm{~h}$ of incubation in normoxic incubator at $37^{\circ} \mathrm{C}$. Cells were then, submitted to different doses of UVB irradiation from a 20 watt MEABS-25 bulb (Ultra-Lum, Inc., Claremont, CA, USA), which emits most of its energy (60\%) with the UVB range $(290-320 \mathrm{~nm})$ with an emission peak at 310 $\mathrm{nm}$ band. The irradiation dosages given were 7 $\mathrm{mJ} / \mathrm{cm}^{2}, 11.5 \mathrm{~mJ} / \mathrm{cm}^{2}$, and $23 \mathrm{~mJ} / \mathrm{cm}^{2}$. After UVB irradiation, cells were returned to the incubator for $5 \mathrm{~h}$ or $24 \mathrm{~h}$ of recovery at $37^{\circ} \mathrm{C}$.

\section{Measurement of the cytotoxicity}

Cell viability was assessed using the Trypan blue exclusion assay as described previously [25]. Briefly, viable and dead cells were discriminated and counted using $0.4 \%$ Trypan blue staining with a hemacytometer (Sigma, Saint-Louis, MO, USA). Dead cells were counted as those stained blue by the Trypan blue stain, whereas live and apoptotic cells were those that had excluded the stain.

\section{Detection of superoxide production}

MSCV-Ht22 and hSelH-Ht22 cells were exposed to a $7 \mathrm{~mJ} / \mathrm{cm}^{2}$ of UVB irradiation in the absence of serum. Production of superoxide anion was assessed using dihydroethidine (HEt) method [31-33] after $5 \mathrm{~h}$ of recovery following $7 \mathrm{~mJ} / \mathrm{cm}^{2}$ of UVB irradiation, because no frank cell death is seen at this time. HEt was purchased from Molecular Probes (Eugene, OR, USA). Stock solution of HEt $(1 \mathrm{mg} / \mathrm{ml})$ was prepared by dissolving in DMSO and stored, protected from light at $-20^{\circ} \mathrm{C}$. One $\mu \mathrm{l}(1 \mu \mathrm{g})$ of HEt was added to $500 \mu \mathrm{l}$ of cultures $15 \mathrm{~min}$ before UVB irradiation. HEt is a cell-permeable fluorescent probe widely used to detect intracellular superoxide anion. It is proposed that the reaction between superoxide and HEt results in the formation of a two-electron oxidized product, which binds to DNA and leads to the enhancement of red fluorescence after being oxidized by superoxide [31, 33, 34]. Twelve microscopic images at the magnification of 
20X were captured and the number of oxidized HEt cells, which represent superoxide production, were counted and presented as percentage of total counted cells. Because HEt gives a light background staining, only cells stained with strong bright red color were counted as oxidized HEt-positive cells.

\section{Statistical analysis}

All experiments were performed at least in triplicate, repeated two to three times and presented as mean \pm SD. ANOVA followed by Scheffe's test was employed for statistical analysis (GraphPad Prism software, SAS, San Diego, CA, USA). A $p$-value of < 0.05 was considered significant.

\section{Results}

\section{hSelH infection}

hSelH was stably infected into Ht22 cells using MSCV vector. The success of this infection was determined by detection of hSelH mRNA levels using quantitative real-time PCR. The mSelH was a low copy number gene in Ht22 cells. The hSelH mRNA level was increased by about 30 folds compared to endogenous mSelH mRNA level in hSelH-Ht22 cells (Table 1). In control MSCV-Ht22 cells, the murine SelH (mSelH) mRNA level, is comparable to that in SelH-Ht22 but the hSelH mRNA is undetectable.

Table 1: Detection of mRNA overexpression on hSelH by Real time PCR. Both human SelH (hselh) and mouse SelH (mselh) genes were measured in hSelH-Ht22 and MSCV-Ht22 cells. Each target gene was amplified in duplicates. The table shows a significant drastic increased level of hSelH in $\mathrm{Ht} 22$-hSelH cells compared to controls (means $\pm \mathrm{SD}, \mathrm{P}<0.0001$ ).

\begin{tabular}{|c|c|c|}
\hline Target genes & MSCV-Ht22 & SelH-Ht22 \\
\hline hSelH & Non-Detectable & $0.557 \pm 0.017^{*}$ \\
mSelH & $0.0149 \pm 0.0019$ & $0.0163 \pm 0.0005$ \\
\hline
\end{tabular}

\section{Effects of variable UVB irradiation duration on cell viability}

In order to identify an optimal time of UVB exposure, we exposed MSCV-Ht22-transfected and hSelH-Ht22-transfected cells to $0 \mathrm{~mJ} / \mathrm{cm}^{2}, 7 \mathrm{~mJ} / \mathrm{cm}^{2}$, $11.5 \mathrm{~mJ} / \mathrm{cm}^{2}$, and $23 \mathrm{~mJ} / \mathrm{cm}^{2}$ of UVB irradiation. Cell viabilities were measured after 24 and 48 h of recovery. Cell viability after $24 \mathrm{~h}$ of recovery, was reduced from $100 \%$ in non-radiated control to an average of $50 \%$, $40 \%$ and $6 \%$ in $7 \mathrm{~mJ} / \mathrm{cm}^{2}, 11.5 \mathrm{~mJ} / \mathrm{cm}^{2}$, and $23 \mathrm{~mJ} / \mathrm{cm}^{2}$ of UVB irradiation, respectively (Figure 1). Cell viabilities were further decreased after $48 \mathrm{~h}$ of recovery. Thus, the average survival rate dropped to $19 \%, 4 \%$ and $0 \%$ following $7 \mathrm{~mJ} / \mathrm{cm}^{2}, 11.5 \mathrm{~mJ} / \mathrm{cm}^{2}$, and 23 $\mathrm{mJ} / \mathrm{cm}^{2}$ of UVB irradiation, respectively (Figure 1). This experiment showed that $7 \mathrm{~mJ} / \mathrm{cm}^{2}$ of UVB irradiation resulted in approximately $50 \%$ cell death at $24 \mathrm{~h}$ of recovery, which was determined to be the optimal length of injury. Therefore, a $7 \mathrm{~mJ} / \mathrm{cm}^{2}$ of UVB irradiation was used for the remaining studies.
Fig. 1. Variable UVB irradiation exposure parameters on cell viability in MSCV (empty vector) transfected Ht22 cells. The irradiation doses given to the cultures were 0 $\mathrm{mJ} / \mathrm{cm}^{2}, 7 \mathrm{~mJ} / \mathrm{cm}^{2}, 11.5 \mathrm{~mJ} / \mathrm{cm}^{2}$, and $23 \mathrm{~mJ} / \mathrm{cm}^{2}$, in DMEM medium with serum, respectively. The percentage of cell viability was determined after 24 and $48 \mathrm{~h}$ of recovery using the Trypan blue exclusion assay. About 100\%, 50\%, 40\% and $6 \%$ of cell viability were observed at $24 \mathrm{~h}$ of recovery 0 $\mathrm{mJ} / \mathrm{cm}^{2}, 7 \mathrm{~mJ} / \mathrm{cm}^{2}, 11.5 \mathrm{~mJ} / \mathrm{cm}^{2}$, and $23 \mathrm{~mJ} / \mathrm{cm}^{2}$ of UVB irradiation, respectively. Cell viabilities were further significantly decreased to $21 \%, 4 \%$ and $0 \%$ at $48 \mathrm{~h}$ of recovery at the indicated UVB doses in the Figure. Experiments were performed in triplicate and presented as means \pm SD. *Significantly different from non-radiated control at $24 \mathrm{~h}(\mathrm{P}<0.05)$ at the indicated UVB dose. \#Significantly different from $24 \mathrm{~h}$ of recovery at $48 \mathrm{~h}$ of recovery $(\mathrm{P}<0.05)$.

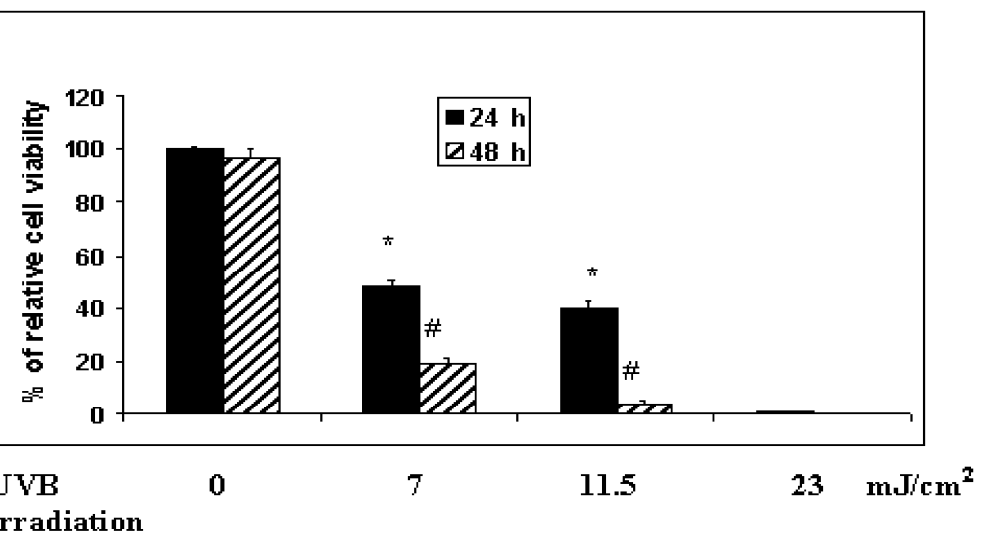

\section{Effects of hSelH overexpression on cell viability after UVB irradiation}

Both MSCV-Ht22 and hSelH-Ht22 cells were exposed to a $7 \mathrm{~mJ} / \mathrm{cm}^{2}$ of UVB irradiation in the presence or absence of serum. The cell viabilities were examined at $24 \mathrm{~h}$ of recovery and the results were compared to MSCV and hSelH transfectants without UVB irradiation. As shown in Figures 2 and 3, MSCV-Ht22 cells exposed to $7 \mathrm{~mJ} / \mathrm{cm}^{2}$ of UVB irradiation in the presence of serum exhibited $48 \%$ cell viability compared to controls, as expected. However, the cell viability was significantly increased in cells overexpressing $\mathrm{hSelH}$. That is, hSelH overexpression resulted in appreciatively $80 \%$ cell survival compared to controls and this was found to be significantly different than that observed with vector control $(\mathrm{P}<0.0001)$. In the absence of serum, cell survival rate decreased to about $70 \%$ even without UVB irradiation, with no major difference between the MSCV-Ht22 and hSelH-Ht22 cells. Cell survival rate was further decreased to $23 \%$ in MSCV-Ht22 cells after a $7 \mathrm{~mJ} / \mathrm{cm}^{2}$ of UVB irradiation. However, with the overexpression of hSelH, cell survival only decreased to $52 \%(\mathrm{P}=$ 0.0004 ) when cells were exposed to $7 \mathrm{~mJ} / \mathrm{cm}^{2}$ of UVB irradiation without supplementation of serum. Figure 2 shows representative photomicrographs taken from MSCV-Ht22 and hSelH-Ht22 cells treated with or without UVB irradiation in the presence or absence of serum. Figure 3 summarizes the cell viability data 
collected from three independent experiments.

Fig. 2. Photomicrographs showing effects of hSelH overexpression cell viability in Ht22 cells treated with 7 $\mathrm{mJ} / \mathrm{cm}^{2}$ of UVB irradiation followed by $24 \mathrm{~h}$ of recovery. Increased cell debris was present in UVB radiated MSCV-Ht22 cell compared to non-treatment control cells. More viable cells were observed when hSelH transfected Ht22 cells were exposed to UVB irradiation. Images were taken at 20X magnification with a Zeiss LSM5 laser-scanning confocal microscope. Bar $=10 \mu \mathrm{m}$.

Fig. 3. Effects of hSelH overexpression on $\mathrm{Ht} 22$ cells exposed to $7 \mathrm{~mJ} / \mathrm{cm}^{2}$ of UVB irradiation with or without serum in media. Cell viability was examined after $24 \mathrm{~h}$ of recovery and presented as percentage (mean \pm SD). Overexpression of hSelH protected cells from UVB-induced damage. *Significantly different from MSCV control, $\mathrm{P}<0.0001$ and \#Significantly different from MSCV transfectant, $\mathrm{P}=0.0004$, in the presence or in the absence of serum, respectively.
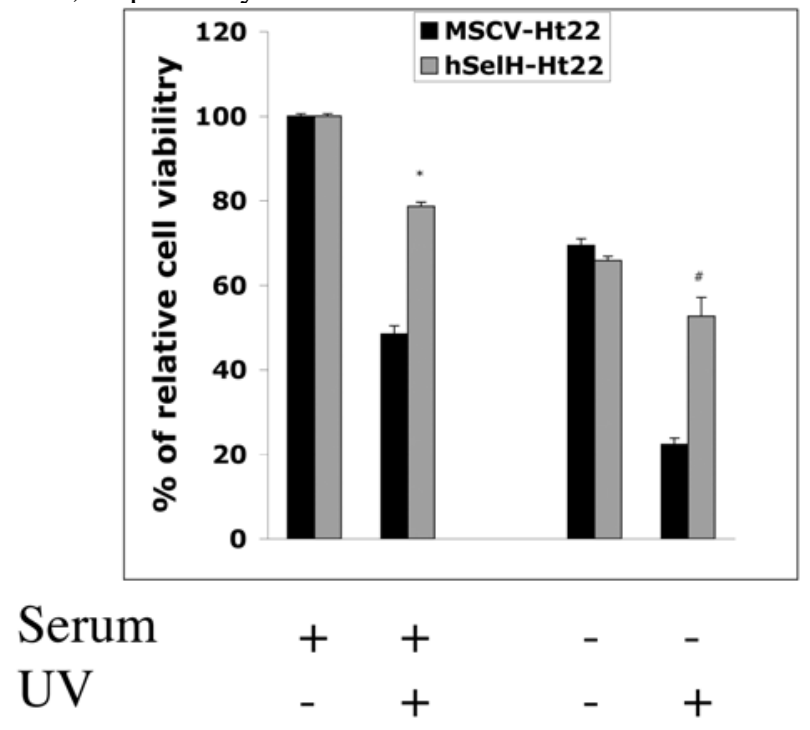

\section{Overexpression of hSelH reduces superoxide formation}

In order to determine the effects of UVB irradiation and overexpression of $\mathrm{hSelH}$ on free radical production, we measured superoxide production using the oxidized HEt method. Since a majority of MSCV-transfected cells die after $24 \mathrm{~h}$ of recovery, we selected an early recovery time point $(5 \mathrm{~h})$ for detection of superoxide formation. A $7 \mathrm{~mJ} / \mathrm{cm}^{2}$ of UVB irradiation resulted in $48 \%$ of oxidized HEt positive stained cells in MSC-Ht22 after $5 \mathrm{~h}$ of recovery. In contrast, the percentage was decreased to $16 \%$ ( $\mathrm{P}=$

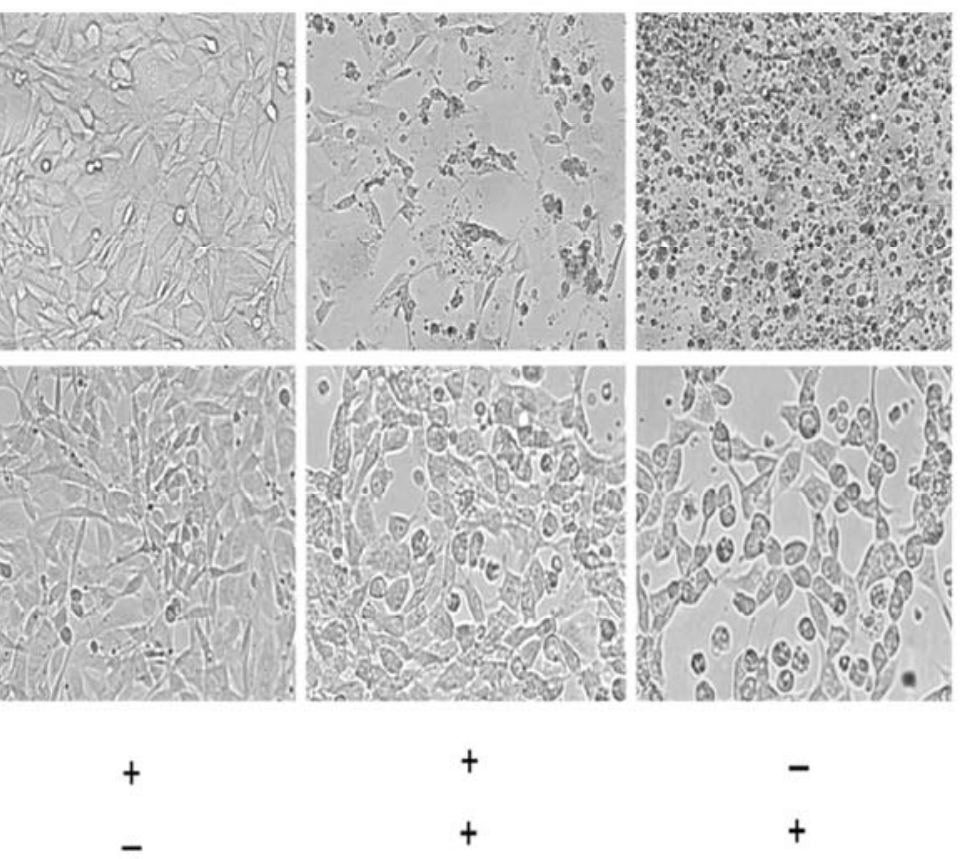

0.0017) in hSelH-Ht22 cells (Figure 4). A set of fluorescent photomicrographs from MSCV-Ht22 and hSelH-Ht22 cells that were exposed to $7 \mathrm{~mJ} / \mathrm{cm}^{2}$ of UVB irradiation is shown in Figure 5.

\section{Discussion}

Previous published studies on neuronal cells have shown that UV irradiation induced apoptosis is associated with activations of capspase, calpain, JNK, and the mitochondrial apoptosis pathway [4-7]. UV irradiation provokes oxidative stress through the generation of ROS and to induce apoptosis by activation of both receptor-mediated and mitochondrion-initiated cell death pathways $[8,9,35$, 38]. In this study, $\mathrm{O} 2-$ production increased significantly after $5 \mathrm{~h}$ of recovery following a $7 \mathrm{~mJ} / \mathrm{cm}^{2}$ of UVB irradiation. This is consistent with the results published by Maglio and colleagues who observed a 5 -fold increase in superoxide production in the skin after UV irradiation [39]. Under physiological conditions, superoxide dismutase (SOD) reduces $\mathrm{O}_{2}$ to $\mathrm{H}_{2} \mathrm{O}_{2}$ that is further reduced to $\mathrm{H}_{2} \mathrm{O}$ by catalase or glutathione peroxidase. However, excess production of $\mathrm{O}^{\circ}$ - leads to formation of the highly toxic hydroxyl radical, $\bullet \mathrm{OH}$, in the presence of metal or peroxynitrite (ONOO-) in the presence of nitric oxide (NO). Hydroxyl radicals and $\mathrm{ONOO}^{-}$are potent oxidants of proteins and DNA, and alter the function of key enzymes through nitration and nitrosylation. Increased ROS in the mitochondrion induces formation of a mitochondrial permeability transition (MPT) pore. The MPT allows release of proapoptotic proteins that subsequently activate a cascade of proteases, ultimately leading to cell death [40,41].

The selenoprotein family contains 25 members in the human proteome [13]. A majority of published studies have been focused on the expression and functions of GPxs, TRs, iodothyronine deiodinases, and SelP. Studies have shown that these enzymes 
possess antioxidative effects, in addition to their roles in male fertility, thyroid hormone metabolism, heavy metal detoxification and neurological function and development [20, 26, 42]. For example, glutathione peroxidase acts as peroxynitrite reductase, preventing oxidation and nitration reactions resulting from peroxynitrate [17, 18]. Similarly, SelP protects low-density lipoproteins (LDL) against oxidation (traps $\mathrm{ONOO}^{-}$and protect astrocytes from various injuries including irradiation and tert-butyl hydroperoxide-induced oxidative stress $(10,21,22,27]$. The functions of most of the recently identified selenoproteins, including $\mathrm{SelH}$, have not been elucidated.

To study whether overexpression of $\mathrm{hSelH}$ is capable of protecting cells against UVB-induced oxidative stress, we first transfected the murine hippocampal Ht22 neuron cell line with the hSelH or the empty vector MSCV. As shown in Table 1, the hSelH mRNA level was 30 fold higher than the endogenous mSelH mRNA level in hSelH-Ht22, suggesting a high transcriptional rate of the transfected hSelH gene. We then exposed the cells to a $7 \mathrm{~mJ} / \mathrm{cm}^{2}$ of UVB irradiation. Overexpression of hSelH reduced cell death caused by UVB irradiation. This result is consistent with a previous publication showing that overexpression of a selenoprotein homologous to glutathione peroxidase blocks UV-induced cell death [43]. We finally measured $\mathrm{O}^{2}$ production in cultured cells at $5 \mathrm{~h}$ after a $7 \mathrm{~mJ} / \mathrm{cm}^{2}$ of UVB irradiation. The results showed that overexpression of hSelH inhibited $\mathrm{O}_{2}{ }^{\circ}$ - formation in neurons in the early recovery phase after irradiation. Concurrently, the percent of oxidized HEt-positive cells detected at $5 \mathrm{~h}$ of recovery was correlated with the percent of cell death observed at $24 \mathrm{~h}$ of recovery, suggesting increased superoxide production might be the underlying cause for the observed cell death. Our findings are in accordance with a previous study reported by Morozova and colleagues [22]. In that study, it was demonstrated that silencing the expression of Drosophila melanogaster SelH (dSelH) gene significantly reduced the total antioxidant capacity and embryo viability. In contrast, overexpression of $\mathrm{dSelH}$ aided cell survival by reducing lipid peroxidation in vivo [28].

It is not clear through what mechanism(s) hSelH reduces ROS production. A parallel study carried by Panee and colleagues has shown that overexpression of SelH sustains the total glutathione (GSH) level in $\mathrm{Ht} 22$ cells during oxidative stress (Panee et al., manuscript in preparation). GSH plays multiple roles in the protection of cells from ROS, electrophiles and xenobiotics [44, 45]. More interestingly, SelH overexpression upregulated the expression of genes involved in antioxidant defense (Panee et al., manuscript in preparation) and phase II detoxification [44]. Therefore, it is likely that SelH overexpression decreases the ROS level and sustains cell viability in Ht22 cells after UVB irradiation via these pathways.

Taken together, our study shows that overexpression of hSelH protects cells against UVB irradiation-induced cell death by ameliorating ROS formation in the early recovery period. The results are in line with previous publications showing that selenoproteins possess antioxidative capacity.

Fig. 4. Percentage of oxidized HEt positive cells in MSCV-Ht22 and hSelH-Ht22 cells after $5 \mathrm{~h}$ of recovery follow a $7 \mathrm{~mJ} / \mathrm{cm}^{2}$ of UVB irradiation. hSelH overexpression significantly reduced oxidized HEt positive cells compared with MSCV-Ht22 cells. *Significantly different from MSCV-Ht22, $\mathrm{P}=0.0017$.

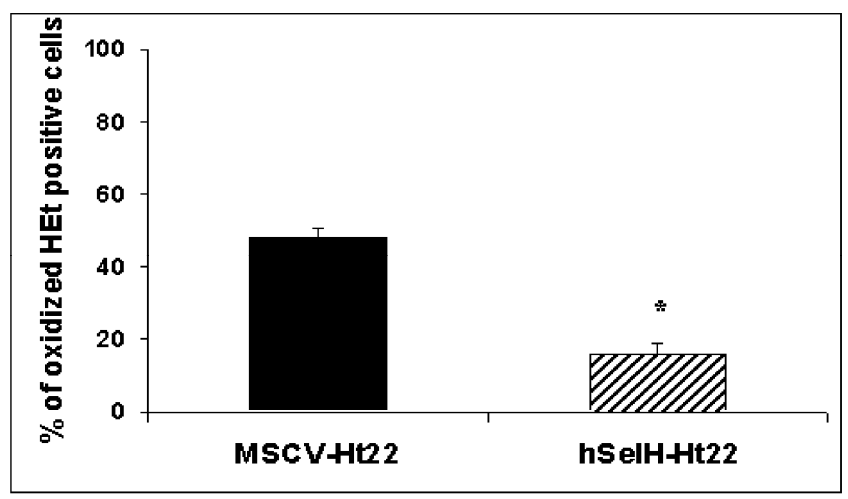

Fig. 5. Representative photomicrographs showing oxidized HEt positive staining in MSCV-Ht22 (A) and hSelH-Ht22 cells (B) after $5 \mathrm{~h}$ of recovery follow 7 $\mathbf{m J} / \mathbf{c m}^{2}$ of UVB irradiation. Arrows denote oxidized HEt cells which indicating the formation of superoxide. Images were captured at $20 \mathrm{X}$ magnification with a Zeiss LSM5 laser-scanning confocal microscope. $\mathrm{Bar}=10 \mu \mathrm{m}$.
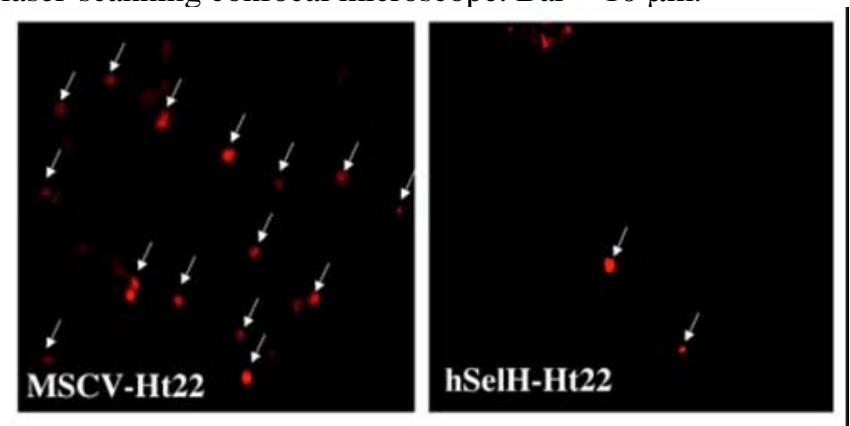

\section{Acknowledgements}

This work was supported in part by $\mathrm{NIH}$ R01NS40302, NIH/G12RR03061, NIH/RR16453, and Hawaii Community Foundation. We thank Sarah Timtim and Andrew Sue-Ako for their active participation in this work during their summer research training, Wanyu Liu for technical assistance, and Drs. Rick Bellinger, Peter Hoffman and Steve Seifried for helpful discussions.

\section{Conflict of interests}

The authors have declared that no conflict of interest exists.

\section{References}

1. Melnikova VO, Ananthaswamy HN. Cellular and molecular events leading to the development of skin cancer. Mutation Res. 2005; 571: 91-106.

2. Licastro F, Sarafian T, Verity AM, Walford RL. Inhibition of 
polymerases-alpha and -beta completely blocks DNA repair induced by uv irradiation in cultured mouse neuronal cells. Biochem Biophys Res Commun. 1985; 132: 929-933.

3. Subrahmanyam K, Rao KS. Ultraviolet light-induced unscheduled DNA-synthesis in isolated neurons of rat brain of different ages. Mech Ageing Dev. 1991; 57: 283-291.

4. Klums D, Schwartz T. Molecular mechanisms of UV-induced apoptosis. Photodermatol Photoimmunol Photomed. 2000; 16: 195-201.

5. McCollum AT, Nasr P, Estus S. Calpain activates caspase-3 during uv-induced neuronal death but only calpain is necessary for death. J Neurochem. 2002; 82: 1208-1220.

6. McCollum AT, Estus S. Ngf acts via p75 low-affinity neurotrophin receptor and calpain inhibition to reduce uv neurotoxicity. J Neurosci Res. 2004; 77: 552-564.

7. Berglund CM, Radesater AC, Persson MA, Budd Haeberlein SL. UV-induced apoptosis in sh-sy5y cells: Contribution to apoptosis by jnk signaling and cytochrome c. J Neurosci Res. 2004; 78: 580-589.

8. Kulms D, Schwartz $\mathrm{T}$. Independent contribution of three different pathways to ultraviolet-B-induced apoptosis. Biochemical Pharmacol. 2002; 64: 837-841.

9. Kulms D, Schwartz T. Molecular mechanisms involved in UV-induced apoptotic cell death. Skin Pharmacol Appl Skin Physiol. 2002; 15: 42-47.

10. Roussyn I, Briviba K, Masumoto H, Sies H. Selenium-containing compounds protect DNA from single-strand breaks caused by peroxynitrite. Arch Biochem Biophys. 1996; 330: 216-218.

11. Burk RF, Hill KE. Selenoprotein P: An extracellular protein with unique physical characteristics and a role in selenium homeostasis. Annu Rev Nutr. 2005; 25: 215-235.

12. Kohrle J. Selenium and the control of thyroid hormone metabolism. Thyroid. 2005; 15: 841-853.

13. Kryukov GV, Castellano S, Novoselov SV, Lebanov AV, Zehtab $\mathrm{O}$, Guigo R, Gladyshev VN. Characterization of mammalian Selenoproteomes. Science. 2003; 300: 1439-1443.

14. Rayman MP. The importance of selenium to human health. Lancet. 2000; 356: 233-241.

15. Saito Y, Takahashi K. Characterization of selenoprotein $\mathrm{P}$ as a selenium supply protein. Eur J Biochem. 2002; 269: 5746-5751.

16. Saito Y, Sato N, Hirashima M, Takebe G, Nagasawa S, Takahashi K. Domain structure of bi-functional selenoprotein P. Biochem J. 2004; 381(Pt 3): 841-846.

17. Sies H, Sharov VS, Klotz LO, Briviba K. Glutathione peroxidase protects against peroxynitrite-mediated oxidations. A new function for selenoproteins as peroxynitrite reductase. J Biol Chem. 1997; 272: 27812-27817.

18. Sies H, Arteel GE. Interaction of peroxynitrite with selenoproteins and glutathione peroxidase mimics. Free Radic Biol Med. 2000; 28: 1451-1455.

19. Hill KE, Zhou J, McMahan WJ, Motley AK, Atkins JF, Gesteland RF, Burk RF. Deletion of selenoprotein P. alters distribution of selenium in the mouse. J Biol Chem 2003; 278: 13640-13646.

20. Hirashima M, Naruse T, Maeda H, Nozaki C, Saito Y, Takahashi $\mathrm{K}$. Identification of selenoprotein $\mathrm{P}$ fragments as a cell-death inhibitory factor. Biol Pharm Bull. 2003; 26: 794-798.

21. Steinbrenner $\mathrm{H}$, Alili L, Bilgic $\mathrm{E}$, Sies $\mathrm{H}$, Brenneisen $\mathrm{P}$. Involvement of selenoprotein $\mathrm{P}$ in protection of human astrocytes from oxidative damage. Free Radic Biol Med. 2006; 40: 1513-1523.

22. Traulsen H, Steinbrenner H, Buchczyk DP, Klotz LO, Sies H. Selenoprotein $\mathrm{P}$ protects low-density lipoprotein against oxidation. Free Radic Res. 2004; 38: 123-128.

23. Burk RF, Hill KE, Awad JA, Morrow JD, Kato T, et al. Pathogenesis of diquat-induced liver necrosis in selenium-deficient rats. Assessment of their roles of lipid peroxidation by measurement of F2 isoprostanes. Hepatology.
1995; 21: 561-569.

24. Valentine WM, Hill KE, Austin LM, Valentine HL, Goldowitz D, Burk RF. Brainstem axonal degeneration in mice with deletion of selenoprotein P. Toxicologic Pathology. 2005; 33: 570-576.

25. Arteel GE, Mostert V, Oubrahim H, Briviba K, Abel J, Sies H. Protection by selenoprotein $\mathrm{P}$ in human plasma against peroxynitrite-mediated oxidation and nitration. Biol Chem. 1998; 379: 1201-1205.

26. Imai H, Nakagawa Y. Biological significance of phospholipid hydroperoxide glutathioneperoxidase (PHGPx, GPx4) in mammalian cells. Free Radic Biol Med. 2003; 34: 145-169.

27. Yant LJ, Ran Q, Rao L, Van Remmen H, Shibatani T, Belter JG, Motta L, Richardson A, Prolla TA. The selenoprotein GPX4 is essential for mouse development and protects from radiation and oxidative damage insults. Free Radic Biol Med. 2003; 34: 496-502.

28. Morozova N, Forry EP, Shahid E, Zavacki AM, Harney JW, Kraytsberg Y, Berry MJ. Antioxidant function of a novel selenoprotein in Drosophila melanogaster. Genes Cells. 2003; 8: 963-971.

29. Muranyi M, Li PA. Hyperglycemia increases superoxide production in the CA1 pyramidal neurons after global cerebral ischemia. Neurosci Lett. 2006; 393: 119-121.

30. Muranyi M, Ding C, He Q, Lin Y, Li PA. Streptozotocin-induced diabetes causes astrocyte death after ischemia and reperfusion injury. Diabetes. 2006; 55: 349-355.

31. Bindokas VP, Jordan J, Lee CC, Miller R. Superoxide production in Rat hippocampal neurons: selective imaging with hydroethidine. J Neurosci. 1996; 16: 1324-1336.

32. Murakami K, Kondo T, Kawase M, Li Y, Sato S, Chen SF, Chan $\mathrm{PH}$. Mitochondrial susceptibility to oxidative stress exacerbates cerebral infarction that follows permanent focal cerebral ischemia in mutant mice with manganese superoxide dismutase deficiency. J Neurosci. 1998; 18: 205-213.

33. Zhao H, Kalivendi S, Zhang H, Joseph J, Nithipatikom K, Vasquez-Vivar J, Kalyanaraman B. Superoxide reacts with hydroethidine but forms a fluorescent product that is distinctly different from ethidium: potential implications in intracellular fluorescence detection of superoxide. Free Radic Biol Med. 2003; 34: 1359-1368.

34. Fridovich I. Editorial commentary on "Superoxide reacts with hydroethidine but forms a fluorescent product that is distinctly different from ethidium: potential implications in intracellular fluorescence detection of superoxide" by $\mathrm{H}$. Zhao et al. Free Radic Biol Med. 2003; 34: 1357-1358.

35. Ho JN, Lee YH, Park JS, Jun WJ, Kim HK, Hong BS, Shin DH, Cho HY. Protective effects of Aucubin isolated from Eucommia ulmoides against UVB-induced oxidative stress in human skin fibroblasts. Biol Pharm Bull. 2005; 128: 244-1248.

36. O'Donovan, $\mathrm{P}$, Perrett CM, Zhang X, Montaner B, Xu Y-Z, Harwood CA, McGregor JM, Walker SL, Hanaoka F, Karran P. Azathioprine and UVA light generate mutagenic oxidative DNA damage. Science. 2005; 306: 1871-1874.

37. Shiu CT, Lee TM. Ultraviolet-B-induced oxidative stress and responses of the ascorbate-glutathione cycle in a marine macroalga Ulva fasciata. J Exp Bot. 2005; 56: 2851-2865.

38. Valencia A, Moran J. Reactive oxygen species induce different cell death mechanisms in cultured neurons. Free Radic Biol Med. 2004; 36: 1112-1215.

39. Maglio DHG, Paz ML, Ferrari A, Weill FS, Czerniczyniec A, Leoni J, Bustamante J. Skin damage and mitochondrial dysfunction after acute ultraviolet B irradiation: relationship with nitric oxide production. Photodermatol Photoimmunol Photomed. 2005; 21: 311-317.

40. Green DR, Reed JC. Mitochondria and apoptosis. Science. 1998; 281: 1309-1312.

41. Green DR, Kroemer G. The pathophysiology of mitochondrial cell death. Science. 2004; 305: 626-629. 
42. Chen J, Berry MJ. Selenium and selenoproteins in the brain and brain diseases. J Neurochem. 2003; 86: 1-12.

43. Shisler JL, Senkevich TG, Berry MJ, Moss B. Ultraviolet-induced cell death blocked by a selenoprotein from a human dermatotropic poxvirus. Science. 1998; 279: 102-151.

44. Lu SC. Regulation of hepatic glutathione synthesis: current concepts and controversies. FASEB J. 1999; 13:1169-1183.

45. Maher P. The effects of stress and aging on glutathione metabolism. Aging Res Rev. 2005; 4: 288-314 